\title{
Using cross-talk simulation to predict the performance of anaglyph 3-D glasses
}

\author{
Andrew J. Woods (SID Member) \\ Chris R. Harris
}

\begin{abstract}
The anaglyph 3-D method is a widely used technique for presenting stereoscopic 3-D images. Its primary advantage is that it will work on any full-color display (LCDs, plasmas, and even prints) and only requires that the user view the anaglyph image using a pair of anaglyph 3-D glasses with usually one lens tinted red and the other lens tinted cyan (blue plus green). A common imagequality problem of anaglyph 3-D images is high levels of cross-talk - the incomplete isolation of the left and right image channels such that each eye sees a "ghost" of the opposite perspective view. An anaglyph cross-talk simulation model has been developed which allows the amount of anaglyph crosstalk to be estimated based on the spectral characteristics of the anaglyph glasses and the display. The model is validated using a visual cross-talk ranking test which indicates good agreement. The model is then used to consider two scenarios for the reduction of cross-talk in anaglyph systems and finds that a considerable reduction is likely to be achieved by using spectrally pure displays. The study also finds that the 3-D performance of commercial anaglyph glasses can be significantly better than handmade anaglyph glasses.
\end{abstract}

Keywords - Stereoscopic, 3-D, cross-talk, ghosting, leakage, anaglyph.

DOI \# 10.1889/JSID20.6.304

\section{Introduction}

The anaglyph 3-D display technique dates back to 1853 when it was developed by William Rollman. ${ }^{1}$ The technique involves the presentation of the left and right perspective images in complementary color channels of the display usually with the left perspective image stored in the red color channel and the right perspective image in the blue and green color channels. To see the anaglyph 3-D image, the observer wears a pair of glasses fitted with color filters in front of each eye - usually red for the left eye and cyan (blue plus green) for the right eye. The color filters act to separate the components of the presented anaglyph 3-D image so that the left perspective image is only seen by the left eye, and the right perspective image is only seen by the right eye, and hence the observer can see a stereoscopic 3-D image.

Anaglyph 3-D has several limitations in terms of the quality of the presented 3-D images - particularly the inability to produce accurate full-color 3-D images (since color is used as the separation or multiplexing technique), binocular rivalry ${ }^{2,3}$ (sometimes known as retinal rivalry) (because each eye sees a different color), and often the presence of high levels of cross-talk (also known as crosstalk or cross talk). ${ }^{4}$ Despite the availability of stereoscopic 3-D display technologies which offer much higher-quality 3-D presentation (e.g., polarized and active shutter glasses), anaglyph continues to be used today in a wide range of applications because it will work with any full-color display and the glasses are very cheap and commonly available, whereas polarized and active shutter 3-D methods require specialized equipment which may not be available to the user. The anaglyph 3-D technique is also seeing high levels of usage because of the current high level of interest in 3-D technologies generally.
Given the continued widespread use of the anaglyph 3-D technique, there is value in efforts to improve the image-quality of this technique. This paper concentrates on the 3-D image quality metric of cross-talk which can be defined as the "incomplete isolation of the left and right image channels" $", 6$ such that one eye can see a ghost image from the other channel. Cross-talk is one of the main determinants of 3-D image quality ${ }^{7}$ and stereoscopic viewing comfort. ${ }^{8,9}$

Although there is very little literature on the perceptual effects of cross-talk in anaglyph 3-D images, there is a good body of work on the perceptual effects of cross-talk in other stereoscopic 3-D display technologies. Cross-talk has been found to "strongly affect subjective ratings of display image quality and visual comfort" in an active-shutter stereoscopic display. ${ }^{10}$ Cross-talk was found to "significantly degrade viewing comfort" in a polarized projected 3-D display. ${ }^{8}$ Cross-talk has also been found to have "a detrimental effect on the perceived magnitude of depth from disparity and monocular occlusions" using a mirror-stereoscope display. ${ }^{11}$ Studies have found cross-talk levels of 5-9\% can significantly affect visual comfort and image quality. 8,10 Our own anecdotal evidence indicates that anaglyph 3-D images are similarly adversely affected by cross-talk.

Several methods have been proposed for improving the perceived quality of anaglyph 3-D images: applying cross-talk cancellation to reduce the perception of ghosting due to cross-talk, ${ }^{12}$ registering the parallax of foreground objects, ${ }^{13}$ using different primary color combinations, ${ }^{14}$ and using different algorithms to calculate the RGB values of the anaglyph image. ${ }^{15-18}$ This paper uses the technique of optimizing the spectral curves of the display and/or glasses

Received 10-18-11; accepted 04-12-12.

The author is with Curtin University, GPO Box U1987, Perth, WA 6845, Australia; telephone +61-8-9266-7920, e-mail: A.Woods@curtin.edu.au. (c) Copyright 2012 Society for Information Display 1071-0922/12/2006-0304\$1.00. 
as a way of reducing anaglyph cross-talk, ${ }^{4,19}$ which is different but complementary to the improvement techniques listed above.

In particular, this paper describes the validation of a cross-talk simulation model which can be used to predict the cross-talk performance of anaglyph 3-D glasses when used with various full-color displays. The availability of an accurate cross-talk simulation model allows a better understanding of anaglyph cross-talk to be gained and also allows the investigation of new techniques which might offer lower cross-talk without needing to perform physical testing.

The test set of anaglyph glasses used in this study provides a good range of cross-talk values over which to validate the cross-talk simulation model (as will be seen in Sec. 4.3). The glasses test set is rather unique in that it can also be used to test another hypothesis. The test set consists of a selection of commercially sourced anaglyph 3-D glasses and also a number of "hand-made" glasses. The hypothesis is that "hand-made" glasses will have inferior 3-D performance compared to that of commercial anaglyph glasses.

Despite the widespread availability of anaglyph 3-D glasses, there will still be circumstances when a user may not have a pair readily available, and to solve this situation there are several sources which recommend constructing a pair of anaglyph 3-D glasses using some simple parts that may be available around the home - notably using colored "cellophane" ${ }^{\prime \prime}$ plastic wrap ${ }^{20-23}$ for the red and cyan filters, or using marker pens ${ }^{24-27}$ and clear plastic sheet to construct the color filters. Anecdotal evidence indicated that handmade anaglyph 3-D glasses would suffer from poor 3-D performance by exhibiting high levels of stereoscopic crosstalk. Visual testing and simulation have been used to verify this hypothesis and validate the cross-talk simulation model.

The analysis is conducted across a broad selection of display devices in order to generalize the results.

\section{Cross-talk simulation}

The cross-talk simulation used in this study builds on past work conducted by the authors and earlier collaborators. $4,14,19$ The program uses spectral data from the displays and glasses in combination with a cross-talk simulation model to estimate the presence of 3-D cross-talk when anaglyph 3-D images presented on emissive full-color displays are viewed using anaglyph 3-D glasses.

The program uses the following cross-talk simulation algorithm:

$$
S_{\mathrm{L}}=\int_{\lambda_{\min }}^{\lambda_{\max }} e(\lambda) g_{\mathrm{L}}(\lambda)\left(m_{L}(\lambda)-b(\lambda)\right) d \lambda
$$

\footnotetext{
${ }^{a}$ Although the term "cellophane" is commonly used to refer to any colored plastic wrap, in many countries it is a registered trademark of Innovia Films, Ltd., UK.
}

$$
\begin{gathered}
S_{\mathrm{R}}=\int_{\lambda_{\min }}^{\lambda_{\max }} e(\lambda) g_{\mathrm{R}}(\lambda)\left(m_{\mathrm{R}}(\lambda)-b(\lambda)\right) d \lambda \\
L_{\mathrm{L}}=\int_{\lambda_{\min } e(\lambda) g_{\mathrm{L}}(\lambda)\left(m_{\mathrm{R}}(\lambda)-b(\lambda)\right) d \lambda}^{\lambda_{\max }} \\
L_{\mathrm{R}}=\int_{\lambda_{\min }}^{\lambda_{\max }}(\lambda) g_{\mathrm{R}}(\lambda)\left(m_{\mathrm{L}}(\lambda)-b(\lambda)\right) d \lambda \\
\mathrm{C}_{\mathrm{L}}=\mathrm{L}_{\mathrm{L}} / \mathrm{S}_{\mathrm{L}} \\
\mathrm{C}_{\mathrm{R}}=\mathrm{L}_{\mathrm{R}} / \mathrm{S}_{\mathrm{R}} \\
\mathrm{C}=\mathrm{C}_{\mathrm{L}}+\mathrm{C}_{\mathrm{R}}
\end{gathered}
$$

where $S$ is the signal intensity (e.g., intensity of the image intended for the left eye as seen at the left eye position, and similar for the right eye); $e$ is the normalized photopic spectral sensitivity of the human eye ${ }^{28}$ as illustrated in Fig. 3(a); $g$ is the spectral transmission of the left or right eye filters of the glasses; $m$ is the emission spectrum of the appropriate color channel(s) of the display monitor; $b$ is the emission spectrum of the black level of the display; $L$ is the leakage intensity (intensity of the image intended for the left eye as seen at the right eye position, or vice versa); $C$ is the crosstalk at each eye (or combined) and usually expressed as a percentage; $\lambda_{\min }$ and $\lambda_{\max }$ describe the wavelength range for the human eye the range of visible light sensitivity is approximately $400-700 \mathrm{~nm}$; and subscripts $\mathrm{L}$ and $\mathrm{R}$ refer to the left-eye channel and right-eye channel, respectively. In a conventional red/cyan anaglyph, $\mathrm{L}$ will refer to the red channel and $\mathrm{R}$ will refer to the cyan (blue plus green) chan-

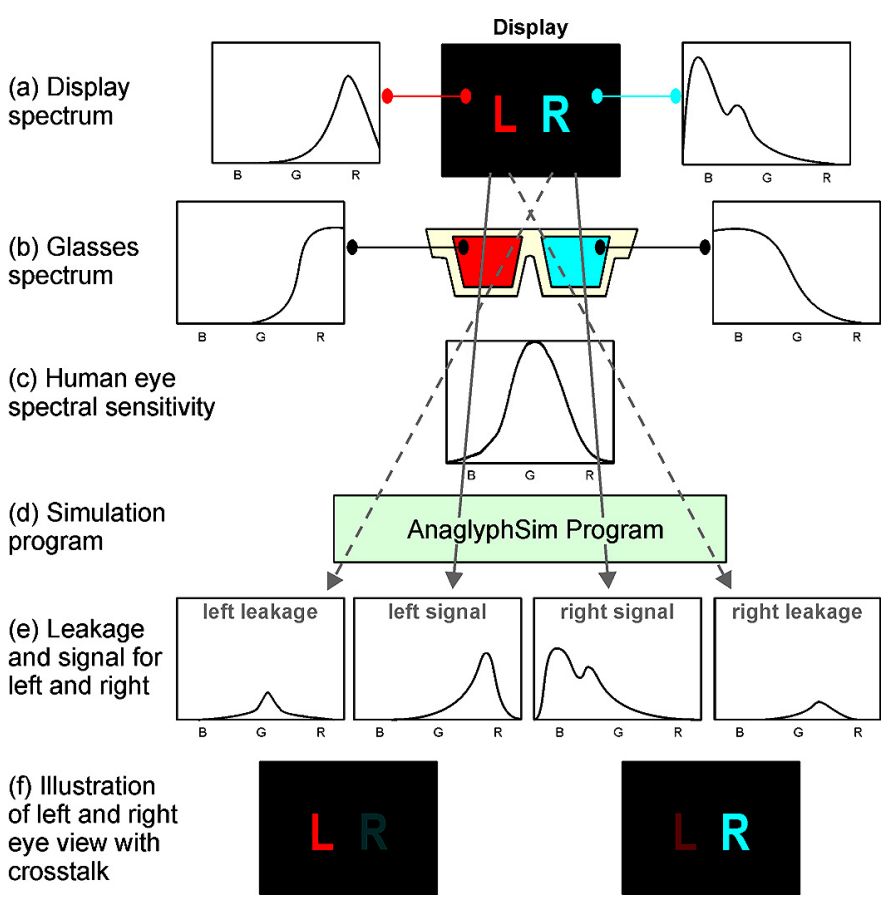

FIGURE 1 - Illustration of the process of anaglyph cross-talk simulation used in this project. 
nel, but other color variations are possible (e.g., blue/yellow or green/magenta ${ }^{14}$ ).

There is no requirement to use a calibrated device to measure $m$ and $b$ - the only requirement is that the same device and scaling is used between measurements. Additionally, $S$ and $L$ can be in arbitrary units because they are only used as a ratio in Eqs. (5) and (6).

This anaglyph cross-talk simulation algorithm is illustrated in Fig. 1 for the example case of a red/cyan anaglyph. Firstly, (a) the emission spectrum of red channel of the display, (b) the spectrum of the red filter of the glasses, and (c) the human-eye spectral sensitivity are multiplied to obtain (e) the spectrum of the intended signal seen by the left eye, and similar for the right eye. The spectrum of the leakage seen by the left eye is obtained by multiplying the spectrum of the blue plus green channels of the display, the spectrum of the red filter of the glasses, and the human eye spectral sensitivity. A similar process is used to determine the righteye leakage. The luminance of each of these signals is obtained by integrating the resulting curves, which is illustrated by the bottom row (f) of this figure. The cross-talk percentage is obtained by dividing the leakage luminance by the signal luminance for each eye as set out in Eqs. (5) and (6). A very similar process would be used if different anaglyph color primaries were used.

The cross-talk performance of anaglyph glasses can vary quite widely from one pair of glasses to another and between different displays. The cross-talk simulation program can very quickly provide an estimate of cross-talk performance across a very large number of combinations of glasses and displays - a process that would be extremely time-consuming and logistically difficult if performed with physical displays and glasses. Another advantage of using a cross-talk simulation program is that it can be used to estimate the cross-talk performance of new or theoretical filters or displays without needing to perform physical testing.

Since the last paper on this topic, ${ }^{14}$ the simulation program, has been updated to use a more recent model of the human-eye spectral sensitivity 28,29 and optimized to significantly increase the speed of operation.

\section{Experimental method}

The cross-talk simulation model was validated using a fourstep process.

\subsection{Spectral emission of displays}

The spectral-emission properties of a selection of displays (LCD, PDP, CRT, and LED DLP $)^{b}$ were measured using an Ocean Optics USB2000 spectroradiometer and also obtained

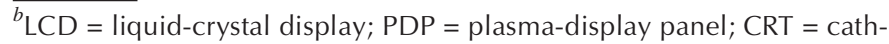
ode-ray tube; $\mathrm{LED}=$ light-emitting diode; $\mathrm{DLP}=$ digital light processing, which uses a digital micro-mirror device (DMD); CCFL = cold-cathode fluorescent lamp.
}

TABLE 1 - Register of tested displays.

\begin{tabular}{|c|l|}
\hline Display ID & \multicolumn{1}{|c|}{$\begin{array}{c}\text { Display Make and Model } \\
\text { (type of display) }\end{array}$} \\
\hline LCD15 & $\begin{array}{l}\text { Samsung 2233RZ } \\
\text { (LCD with CCFL backlight) }\end{array}$ \\
\hline PDP15 & $\begin{array}{l}\text { Samsung PS50A450 } \\
\text { (Plasma Display Panel) }\end{array}$ \\
\hline CRT20 & $\begin{array}{l}\text { Mitsubishi Diamond View 1771ie } \\
\text { (Cathode Ray Tube) }\end{array}$ \\
\hline LEDDLP1 & $\begin{array}{l}\text { Samsung HLT5689 } \\
\text { (rear projection DLP using an LED light engine) }\end{array}$ \\
\hline
\end{tabular}

from previous studies. ${ }^{4,14}$ Table 1 lists the displays used in this study.

The "Glasses IDs" and "Display IDs" used here correspond to the identification series first started in Ref. 19 and continued in Refs. 14 and 4 and are consistent among these studies.

It should be noted that particular care must be exercised when measuring the spectrum of the displays in order to minimize measurement error due to the measurement technique. In the case of the PDP and CRT monitors, their impulse-type operation can create synchronization issues with the sampling period of the sensor. Although all of the tested displays have some time-varying light output, PDP and CRT have the most variation. Long integration times should be used to minimize the effect of the time-varying light output. In the case of PDPs, another factor to consider is the presence of an automatic brightness limiter (ABL) which reduces the intensity of high-brightness scenes (to reduce power consumption). Full-screen test charts should not be used in order to avoid triggering the ABL, which would otherwise affect the measurement of the relative balance of the red, green, and blue color channels. The test charts should therefore be limited to a small portion of the screen, against a black background. The sensing head of the spectroradiometer should also not be placed too close to the surface of the screen such that the spatial separation of the color subpixels would be detected by the sensor.

\subsection{Spectral transmission of glasses}

The 12 pairs of anaglyph glasses tested in this study are listed in Table 2. The selection of glasses consists of three commercial pairs, three pairs constructed using marker pens, and six pairs constructed using colored plastic wrap ("cellophane"). This selection of glasses provided a wide range of cross-talk values which was useful for validating the cross-talk simulation model.

The three pairs of marker pen anaglyph glasses were constructed by using red/blue pairs of marker pens purchased from retail outlets. The marker pens were used to draw red and blue filter samples on a fresh sheet of overhead transparency film. The overhead transparency film used had good clarity and optical performance, in keeping with its manufacture for use in an optical projection application. 
TABLE 2 - Register of anaglyph glasses tested in this study.

\begin{tabular}{|l|l|}
\hline $\begin{array}{l}\text { Glasses } \\
\text { ID }\end{array}$ & \multicolumn{1}{c|}{ Description } \\
\hline Commercial anaglyph glasses \\
\hline 3DG73 & NVIDIA 3D Vision Discover \\
\hline 3DG74 & $\begin{array}{l}\text { Stereoscopic Displays and Applications 2006 - } \\
\text { manufactured by American Paper Optics }\end{array}$ \\
\hline 3DG88 & Top Gear - manufactured by OZ3D Optics \\
\hline Hand-made marker-pen anaglyph glasses \\
\hline 3DG77 & $\begin{array}{l}\text { "hand-drawn" using Sharpie Fine Point } \\
\text { Permanent Marker- red and blue (on clear } \\
\text { overhead transparency film) }\end{array}$ \\
\hline 3DG78 & $\begin{array}{l}\text { "hand-drawn" using Artline 70 - red and blue (on } \\
\text { clear overhead transparency film) }\end{array}$ \\
\hline 3DG79 & $\begin{array}{l}\text { "hand-drawn" using Artline 854 OHP Permanent } \\
\text { Marker - red and blue (on clear overhead } \\
\text { transparency film) }\end{array}$ \\
\hline Hand-made 'Cellophane' anaglyph glasses \\
\hline 3DG80 & John Sands "Plain Cello" - red and blue \\
\hline 3DG81 & $\begin{array}{l}\text { John Sands "Plain Cello" (two layers) - red and } \\
\text { blue }\end{array}$ \\
\hline 3DG82 & Henderson Greetings "cello" - red and blue \\
\hline 3DG83 & $\begin{array}{l}\text { Henderson Greetings "cello" (two layers) - red } \\
\text { and blue }\end{array}$ \\
\hline 3DG84 & Unbranded "clear wrap" - red and blue \\
\hline 3DG85 & $\begin{array}{l}\text { Unbranded "clear wrap" (two layers) - red and } \\
\text { blue }\end{array}$ \\
\hline
\end{tabular}

The "cellophane" glasses were constructed from three different brands of red and blue sets of colored plastic wrap purchased at retail outlets. Each brand of wrap was used to construct two pairs of anaglyph glasses; firstly, with a single layer of plastic film in each eye (red in one eye and blue/cyan in the other eye), and, secondly, with two layers of the plastic wrap.

The optical spectral transmission of the anaglyph filters were measured with a Perkin Elmer Lambda 35 spectrophotometer.

It should be noted that some of the hand-made glasses have some non-ideal optical properties other than their spectral transmission performance - specifically, the clarity of the lens [which degrades the modulation transfer function (MTF)], dispersion, and variability of the ink density. The marker-pens tend to have a considerable amount of variability of ink density (across the filter and from filter to filter) due to the manual way in which the ink is applied. Glasses 3DG81, 3DG84, and 3DG85 have the worst clarity of all the glasses making the image soft focused.

\subsection{Cross-talk simulation}

The spectral data from the displays and glasses was processed using the anaglyph cross-talk simulation program described in Sec. 2. This provides a cross-talk percentage estimate for both filters of every pair of glasses when used with each display - in this particular project a total of 96 values.

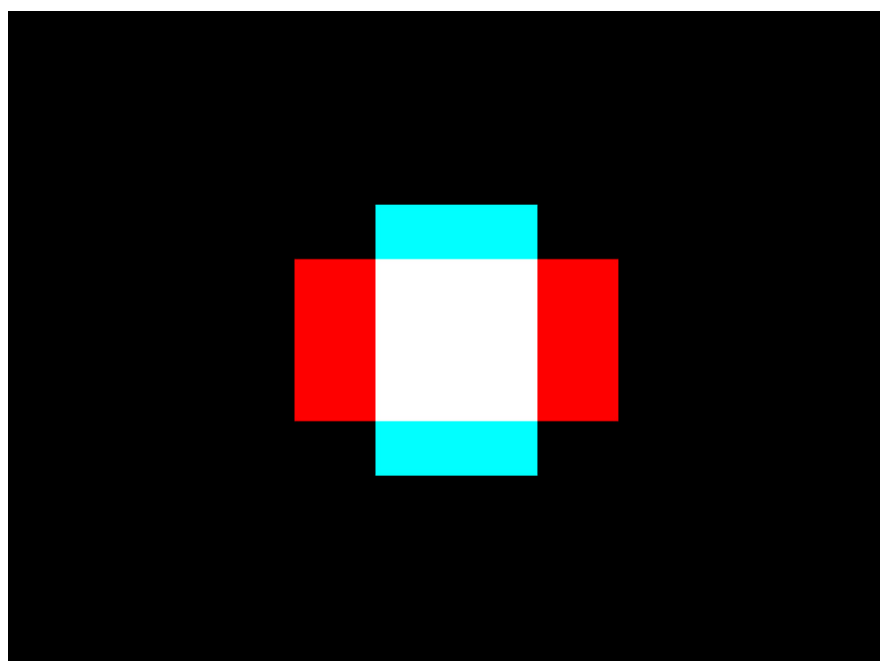

FIGURE 2 - The visual test target used during the anaglyph cross-talk visual ranking tests.

\subsection{Visual ranking}

The cross-talk performance of the various anaglyph filters were visually ranked to allow a comparison with the crosstalk simulation results. The glasses listed in Table 2 were mounted in similar white frames, ordered randomly, and each observer was asked to rank the glasses from lowest cross-talk to highest cross-talk whilst looking at the test graphic (see Fig. 2) presented on each target display (from Table 1). Five observers (labeled Ob1 to Ob5) took part in the visual ranking tests. Each observer was provided with a randomly ordered stack of glasses. The observers were asked to compare two glasses at a time using the test graphic and to place the glasses on the table in front of them with the lowest cross-talk glasses on the left to the highest crosstalk on the right. Each observer made multiple passes through the set of glasses in front of them, comparing two glasses at a time using the test graphic, to sort the glasses into the correct order, and finally confirm that the glasses were in the correct order. Each observer performed separate sorting tasks for the red and cyan filters across each of the four displays, so that each observer performed eight sorting tasks. The room was dimly lit to reduce the likelihood of ambient light or frame luminance affecting the results. ${ }^{30}$

The visual validation test was conducted on the basis of the relative ranking of the cross-talk performance of the glasses because the human-visual system is not accurate at determining absolute measurement of brightness (known as "lightness constancy"), ${ }^{31}$ whereas the human-visual system is usually very good at performing relative brightness comparisons.

The test target used in this study (Fig. 2) allows two types of cross-talk comparison to be performed. In the case of a validation test with the red filters: Firstly, the relative brightness of the leaked cyan rectangle relative to the brightness of the passed red rectangle will give one indication of the cross-talk level, and, secondly, the relative brightness of the center white square relative to the brightness of the passed red rectangle will also give an indication of cross- 
(a) Human Eye Spectral Sensitivity

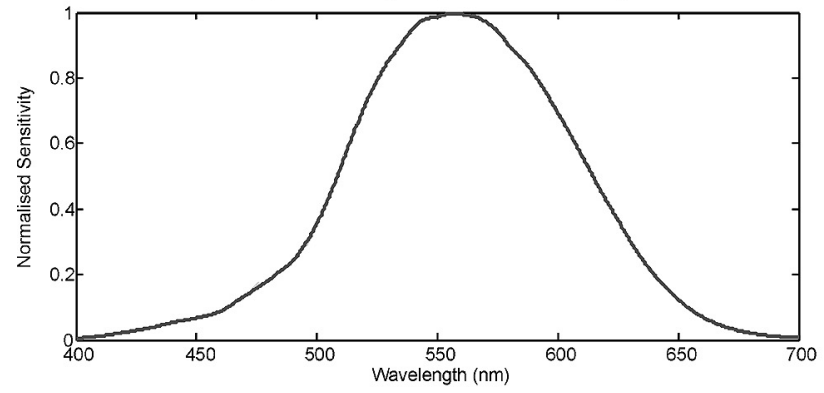

(b) Display Emission - Red Channel

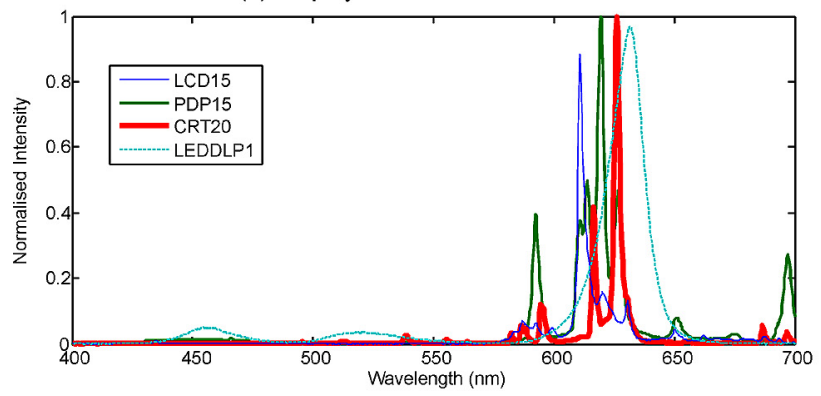

(e) Red Filter of 'Cellophane' Glasses

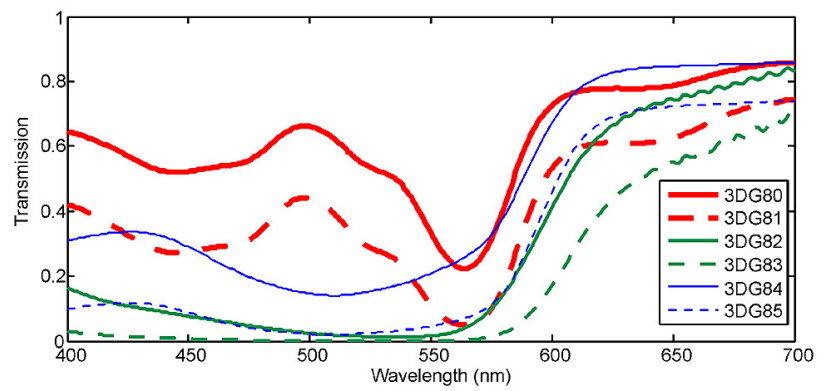

(g) Red Filter of Commercial and Marker-Pen Glasses

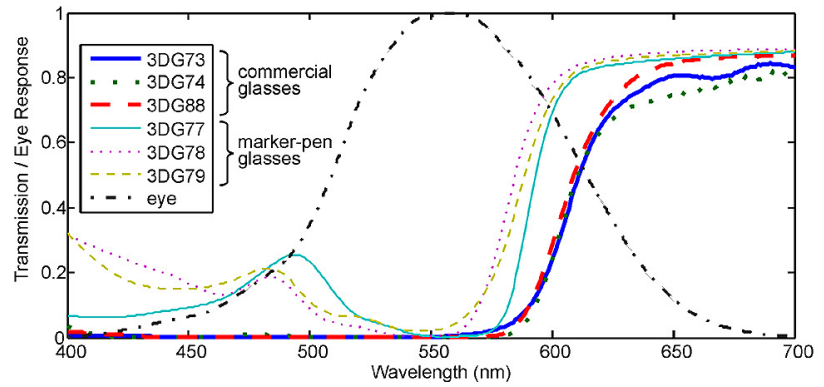

(c) Display Emission - Green Channel

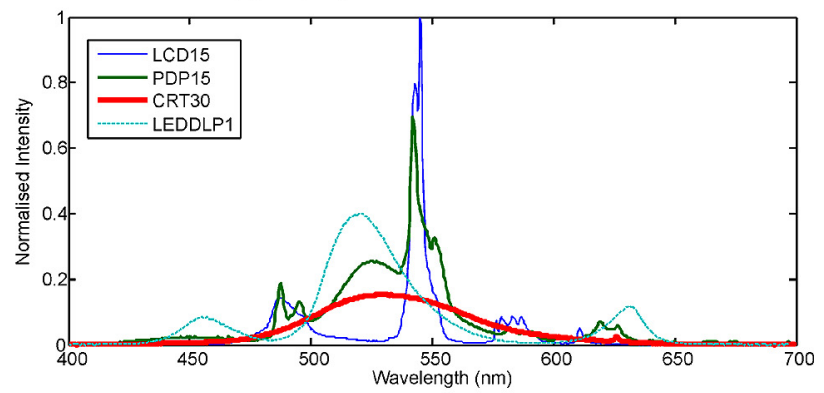

(d) Display Emission - Blue Channel

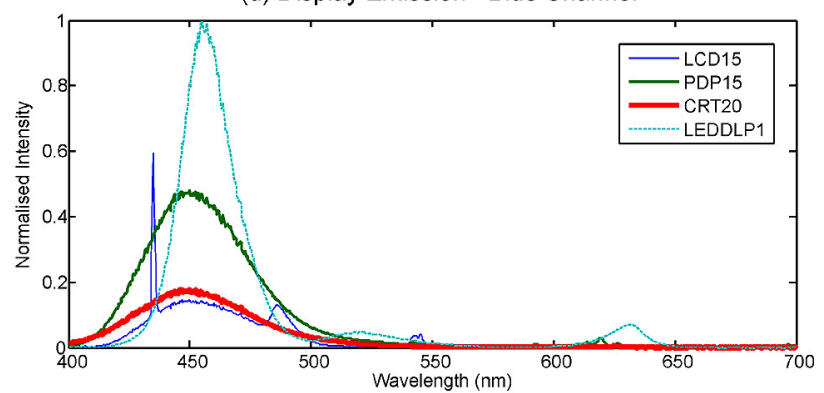

(f) Cyan Filter of 'Cellophane' Glasses

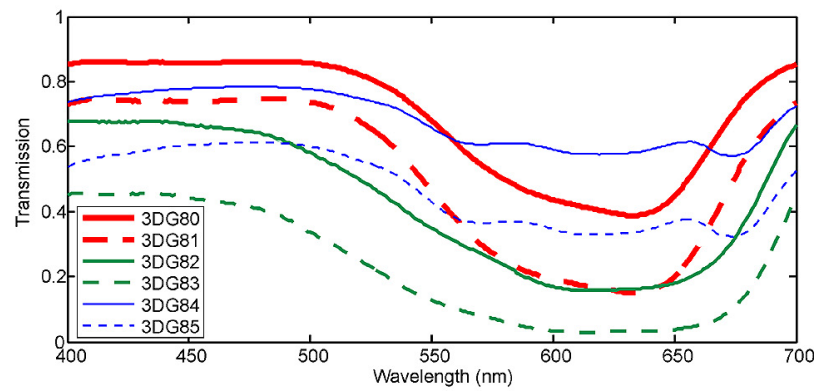

(h) Cyan Filter of Commercial and Marker-Pen Glasses

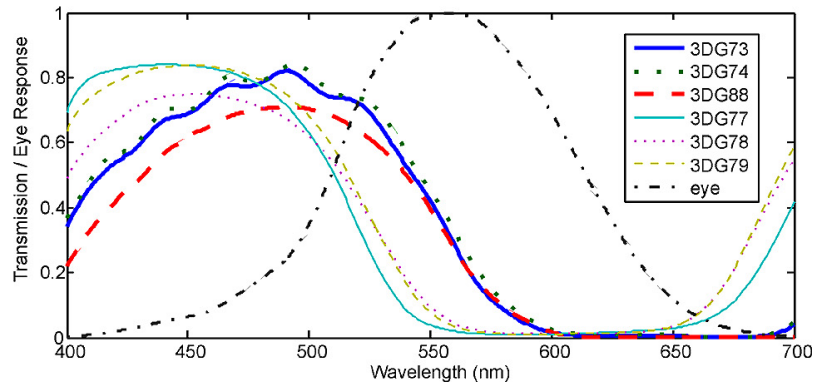

FIGURE 3 - Spectral plots of (a) human-eye spectral sensitivity, (b) the emission spectrum of the red channel of the tested displays, (c) the emission spectrum of the green channel of the tested displays, (d) the emission spectrum of the blue channel of the tested displays, (e) red filter of the tested "cellophane" glasses, ( $\mathrm{f}$ ) cyan filter of the tested "cellophane" glasses, (g) red filter of the commercial and marker-pen glasses with the human-eye response also indicated, and (h) cyan filter of the commercial and marker-pen glasses with human-eye response also indicated. The plots are shown vertically stacked with the same horizontal axis to allow easy comparison between different plots of the same color range.

talk level. It is usually easier to use the first method to compare glasses with low cross-talk levels and the second method for mid-to-high levels of cross-talk. The observers were briefed accordingly, but were free to use whichever method they found easiest.

The observers were asked to try to only consider crosstalk differences between the glasses and ignore other optical differences such as relative brightness, relative clarity, and variability of the filter pigments. The marker pen filters usually had a high level of pigment variability. Some of the "cellophane" filters had very poor clarity and softened the image considerably. Several of the observers commented that it was difficult to compare cross-talk levels between two filters which had vastly different clarity, particularly when the cross-talk levels were seemingly close, which may lead to ranking error. 


\section{$4 \quad$ Results}

\subsection{Display spectra}

The spectra of the four sampled emissive displays are shown in Figs. 3(b)-3(d) for each of the three color channels. The three curves for each display have been scaled such that the maximum of the three curves for each display is normalized to one. It can be seen that there is a considerable variation between the spectral curves of different displays for each color primary. This is due to each of the displays using very different light-generation and modulation techniques.

When considering the anaglyph performance of various emissive displays, of key importance is the amount of light emitted in the "out of band" areas for each color channel. For example, a green color primary would ideally only emit light in the approximate range $500-570 \mathrm{~nm}$, but as can be seen in Fig. 3(c), most of the displays output a significant amount of light outside this range. More light output in the out-of-band areas for each color channel will contribute to higher levels of anaglyph cross-talk - this is considered further in Sec. 4.3.

\subsection{Glasses spectral transmission}

The spectral transmission of the glasses tested in this study are shown in Figs. 3(e)-3(h). The spectral transmission of the hand-made "cellophane" glasses are shown in Figs. 3(e) and $3(\mathrm{f})$. The spectral transmission of the commercial anaglyph glasses and the hand-made marker-pen glasses are shown in Figs. 3(g) and 3(h).

The spectral performance limitations of the "cellophane" glasses are clearly evident in Figs. 3(e) and 3(f). In an ideal pair of anaglyph glasses, the filters should pass the intended color band and block the unwanted color bands, with the blocking of the unwanted channels being the most important. For example, with a red filter, it should pass the red part of the spectrum (roughly 590-700 nm) and block the blue and green parts of the spectrum (roughly 400-570 $\mathrm{nm}$ ). With most of the "cellophane" glasses, it can be seen that the unwanted color ranges are not well attenuated. Referring to the plots of the red filter of 3DG80, 3DG81, and 3DG84 in Fig. 3(e), it can be seen that these filters do not provide very much attenuation of wavelengths from 400 to $570 \mathrm{~nm}$ (the blue and green areas of the visible spectrum) which will result in significant leakage and therefore high cross-talk. This can be compared with the spectral performance of the red commercial filter 3DG88 in Fig. 3(g), which has very low transmission in the blue-green wavelength range. The marker-pen filters shown in Figs. 3(e) and 3(f) also show a similar insufficient attenuation in the 400-570$\mathrm{nm}$ range which will also point to poor cross-talk performance. The cross-talk performance of the glasses will be discussed further from a simulation standpoint below. (a) Red Filters

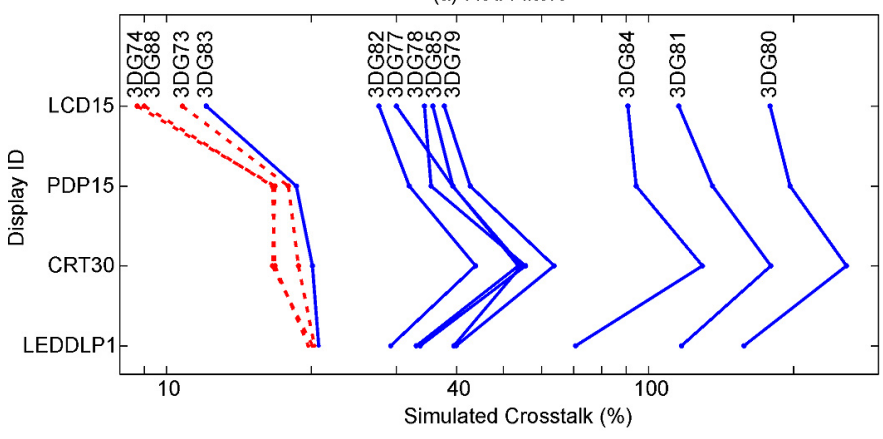

(b) Cyan Filters

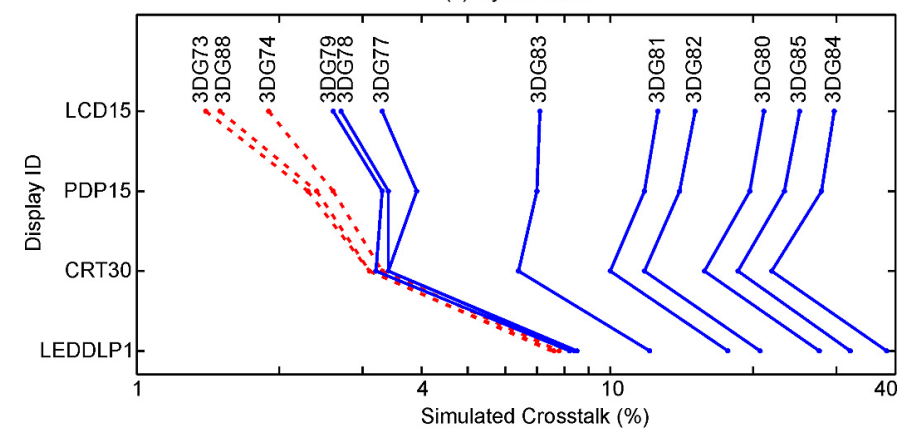

FIGURE 4 - Illustration of the results of the cross-talk simulation of the 12 sets of glasses across the four tested displays for (a) red filters and (b) cyan filters. The commercial anaglyph glasses are plotted in dashed red.

\subsection{Cross-talk simulation}

The cross-talk simulation program results for the 12 sets of anaglyph glasses (three commercial pairs and nine handmade pairs) are shown in Table 3 for each of the four displays. The simulation program calculates the cross-talk for the left and right eyes separately, as shown in the table, and in addition provides an estimate of overall cross-talk (the sum of the cross-talk value from the left and right eyes). Table 3 has been sorted from lowest mean overall cross-talk to highest mean overall cross-talk.

The cross-talk simulation program results for the separate red and cyan filters for each display are also illustrated in Fig. 4. This figure allows an inter-display comparison of the relative performance of the different filters across different displays to be easily seen. The horizontal axis of both of these plots is shown on a logarithmic scale because it reduces the bunching of the results on the left-hand side of the plots, and the human-visual response has been described as having a logarithmic-like response to light over a limited range. 32,33

With reference to Fig. 4, it can be seen that the rank order of the simulated cross-talk of the tested filters is mostly the same from one display to another as illustrated by the mostly non-intersecting line segments. A few crossovers do occur, and these will be caused by the differences between the shapes of the spectral curves of the different displays and the way these interact with the different shaped spectral curves of the filters.

With only a few exceptions, the simulation predicts that the commercial anaglyph filters will offer substantially 
TABLE 3 - Cross-talk calculation results of the four displays The lowest "overall cross-talk" for each display has been highlighted in rich green. "Overall cross-talk" of less than 15 has been highlighted in light green. The highest "overall cross-talk" for each display has been highlighted in orange.

\begin{tabular}{|c|c|c|c|c|c|c|}
\hline \multirow{2}{*}{\multicolumn{2}{|c|}{$\begin{array}{r}\text { Crosstalk (\%) } \\
\text { Glasses } \\
\end{array}$}} & \multicolumn{4}{|c|}{ Displays } & \multirow[b]{2}{*}{ mean } \\
\hline & & \multirow{2}{*}{$\frac{\text { LCD15 }}{9.0}$} & \multirow{2}{*}{$\frac{\text { PDP15 }}{16.6}$} & CRT30 & $\begin{array}{c}\text { LED } \\
\text { DLP1 } \\
\end{array}$ & \\
\hline & Red & & & 16.8 & 19.7 & \\
\hline 3DG88 & Cyan & 1.5 & 2.4 & 3.1 & 7.5 & \\
\hline commercial & Overall & 10.5 & 19.0 & 19.9 & 27.2 & 19.1 \\
\hline & Red & 8.7 & 16.8 & 16.6 & 20.1 & \\
\hline 3DG74 & Cyan & 1.9 & 2.6 & 3.3 & 7.8 & \\
\hline commercial & Overall & 10.6 & 19.5 & 19.9 & 27.9 & 19.5 \\
\hline & Red & 10.8 & 17.9 & 18.8 & 20.3 & \\
\hline 3DG73 & Cyan & 1.4 & 2.3 & 3.1 & 7.6 & \\
\hline commercial & Overall & 12.2 & 20.2 & 21.9 & 27.9 & 20.5 \\
\hline & Red & 12.1 & 18.6 & 20.1 & 20.7 & \\
\hline 3DG83 & Cyan & 7.1 & 7.0 & 6.4 & 12.1 & \\
\hline 'cellophane' & Overall & 19.2 & 25.6 & 26.5 & 32.8 & 26.0 \\
\hline & Red & 34.3 & 35.4 & 55.6 & 33.6 & \\
\hline 3DG78 & Cyan & 2.7 & 3.4 & 3.4 & 8.4 & \\
\hline marker-pen & Overall & 37.0 & 38.8 & 58.9 & 42.0 & 44.2 \\
\hline & Red & 30.0 & 39.2 & 54.5 & 39.5 & \\
\hline 3DG77 & Cyan & 3.3 & 3.9 & 3.4 & 8.5 & \\
\hline marker-pen & Overall & 33.3 & 43.1 & 57.9 & 48.0 & 45.6 \\
\hline & Red & 27.6 & 31.9 & 43.8 & 29.2 & \\
\hline 3DG82 & Cyan & 15.1 & 14.0 & 11.8 & 20.7 & \\
\hline 'cellophane' & Overall & 42.7 & 45.9 & 55.6 & 49.9 & 48.5 \\
\hline & Red & 37.7 & 42.7 & 63.7 & 39.9 & \\
\hline 3DG79 & Cyan & 2.6 & 3.3 & 3.2 & 8.2 & \\
\hline marker-pen & Overall & 40.3 & 46.0 & 66.9 & 48.1 & 50.3 \\
\hline & Red & 35.7 & 39.3 & 53.7 & 33.0 & \\
\hline 3DG85 & Cyan & 25.1 & 23.3 & 18.6 & 32.1 & \\
\hline 'cellophane' & Overall & 60.8 & 62.6 & 72.4 & 65.0 & 65.2 \\
\hline & Red & 90.6 & 94.3 & 129.4 & 70.6 & \\
\hline 3DG84 & Cyan & 29.7 & 27.9 & 21.9 & 38.3 & \\
\hline 'cellophane' & Overall & 120.3 & 122.2 & 151.3 & 108.9 & 125.7 \\
\hline & Red & 115.5 & 135.8 & 179.4 & 117.1 & \\
\hline 3DG81 & Cyan & 12.6 & 11.8 & 10.0 & 17.7 & \\
\hline 'cellophane' & Overall & 128.1 & 147.6 & 189.3 & 134.8 & 149.9 \\
\hline & Red & 178.7 & 196.7 & 257.3 & 157.8 & \\
\hline 3DG80 & Cyan & 21.1 & 19.7 & 15.8 & 27.6 & \\
\hline 'cellophane' & Overall & 199.8 & 216.4 & 273.1 & 185.4 & 218.7 \\
\hline
\end{tabular}

lower cross-talk than the other filters. With the better performing glasses (the commercial glasses), the simulation also points to some big differences in cross-talk performance from one display to another - for example, the simulation predicts that the commercial glasses will provide much lower cross-talk when used with LCD15 than the other displays, for both filter colors.

The simulation also predicts a good spread in the cross-talk performance of the selection of test filters used in this study - which in turn will aid in the validation of the simulation algorithm.
Some of the cross-talk simulation values presented in Table 3 are greater than $100 \%$ (i.e., the worst performing filters) - the reader might at first think this is impossible, but this can occur with anaglyph cross-talk because the blue and green channels combined (one eye) have a much higher luminance than the red channel (the other eye).

It can be seen from Fig. 4(a) that the red filter of 3DG83 has a predicted cross-talk performance very close to that of the commercial filters; however, the cyan filter of 3DG83 has quite poor predicted cross-talk performance. Additionally, both of these marker-pen ink filters have high ink-density variability which degrade the visual quality of the glasses as a whole.

\subsection{Visual ranking and validation}

The visual ranking experiment involved 40 separate crosstalk ranking tasks across five observers, 12 pairs of glasses (two filters in each pair of glasses), and four different displays, resulting in 480 separate observations. The results of the visual ranking experiment are illustrated in Fig. 5. The glasses ranking results for each display, observer, and filter color combination are plotted against the corresponding simulated cross-talk ranking for that display and filter color. A line segment joins the visual ranking with the simulated ranking for each pair of glasses.

When plotting the ranking results, we had the option of showing the ranking observations with an equal spacing between observations; however, this would give an unrealistic equal visual emphasis on ranking observations regardless of how close or disparate the cross-talk is between those particular filters. We therefore decided to plot the results on horizontal axis values which correspond to the simulated

TABLE 4 - Example of the ranking representation technique used in Fig. 5 for Observer 2 ranking the cyan filters on LEDDLP1.

\begin{tabular}{|c|c|c|c|}
\hline $\begin{array}{c}\text { simulated } \\
\text { rank order }\end{array}$ & $\begin{array}{c}\text { simulated rank } \\
\text { rank order on } \\
\text { visual } \\
\text { simulated } \\
\text { crosstalk scale } \\
(\%)\end{array}$ & $\begin{array}{c}\text { visual rank } \\
\text { order on } \\
\text { simulated } \\
\text { crosstalk scale } \\
(\%)\end{array}$ \\
\hline 1 & 1 & 7.5 & 7.5 \\
\hline 2 & 6 & 7.6 & 8.5 \\
\hline 3 & 5 & 7.8 & 8.4 \\
\hline 4 & 4 & 8.2 & 8.2 \\
\hline 5 & 3 & 8.4 & 7.8 \\
\hline 6 & 2 & 8.5 & 7.6 \\
\hline 7 & 7 & 12.1 & 12.1 \\
\hline 8 & 8 & 17.7 & 17.7 \\
\hline 9 & 9 & 20.7 & 20.7 \\
\hline 10 & 11 & 27.6 & 32.1 \\
\hline 11 & 10 & 32.1 & 27.6 \\
\hline 12 & 12 & 38.3 & 38.3 \\
\hline
\end{tabular}


(a) LCD15 Red

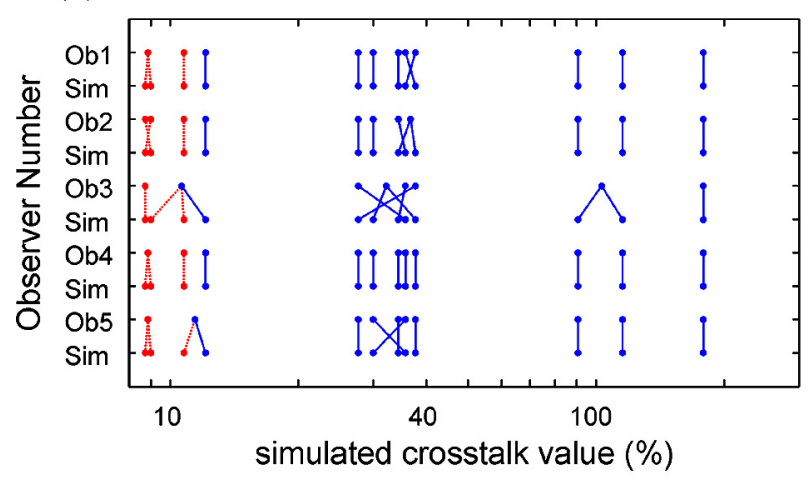

(c)

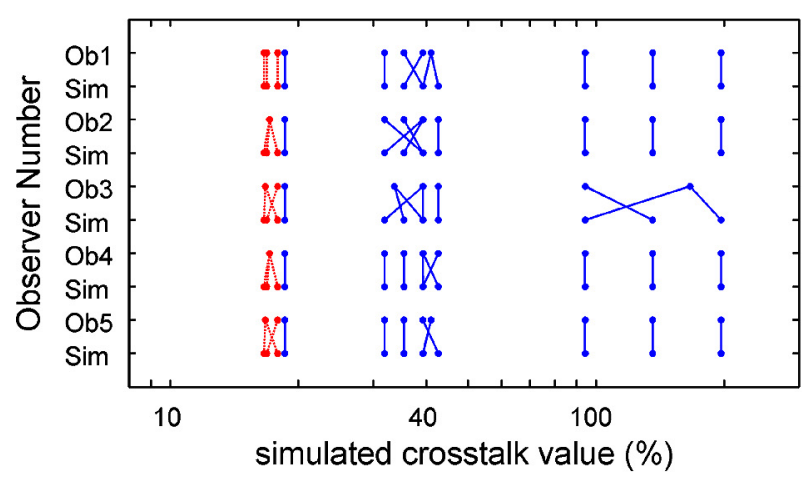

(e)

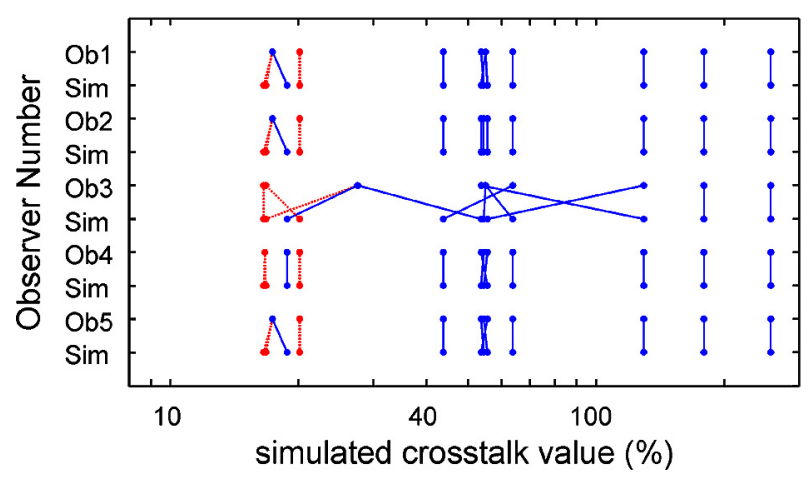

(g)

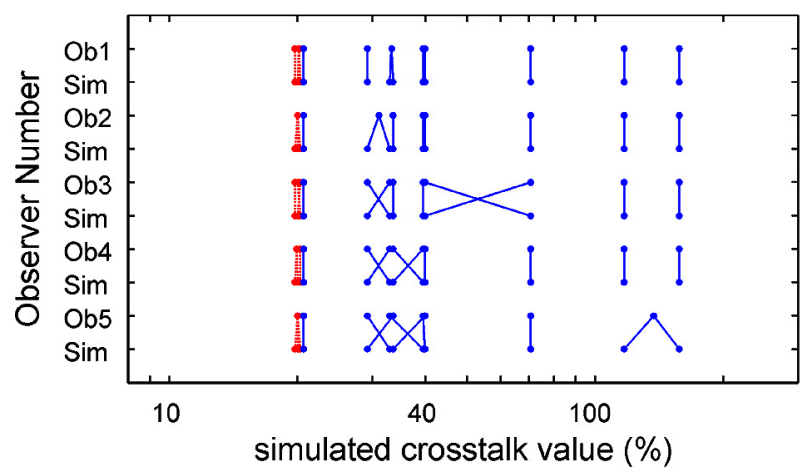

(b) LCD15 Cyan

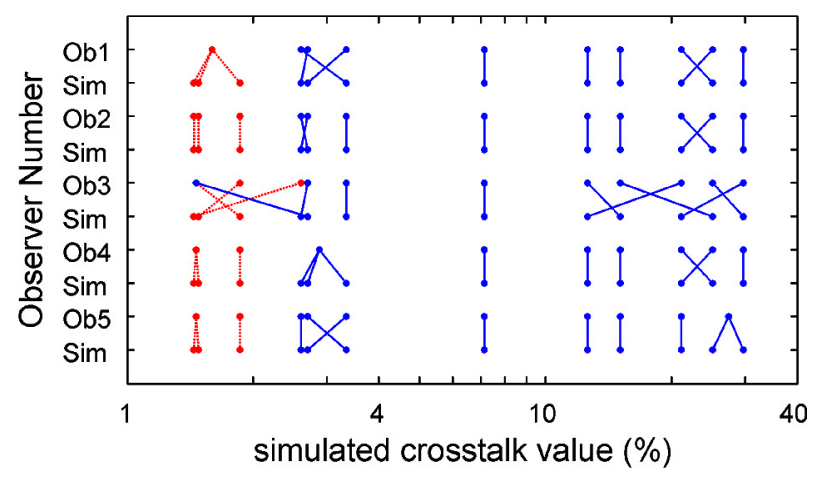

(d)

PDP15 Cyan

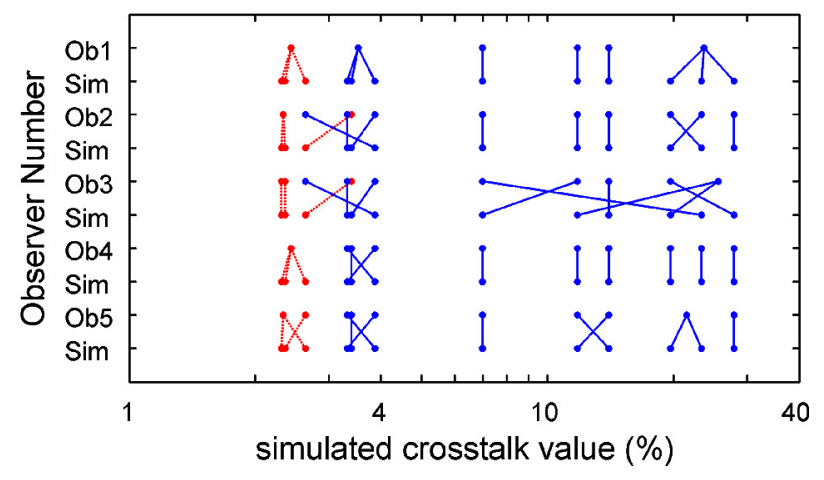

(f)

CRT30 Cyan

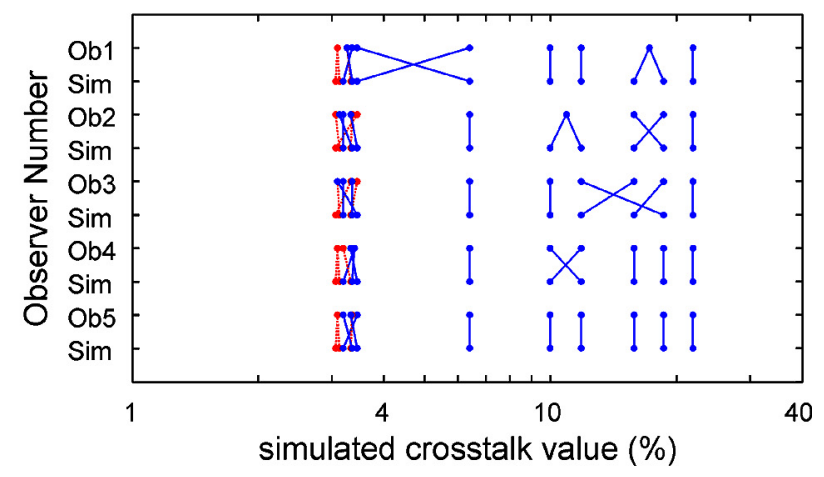

(h) LEDDLP1 Cyan

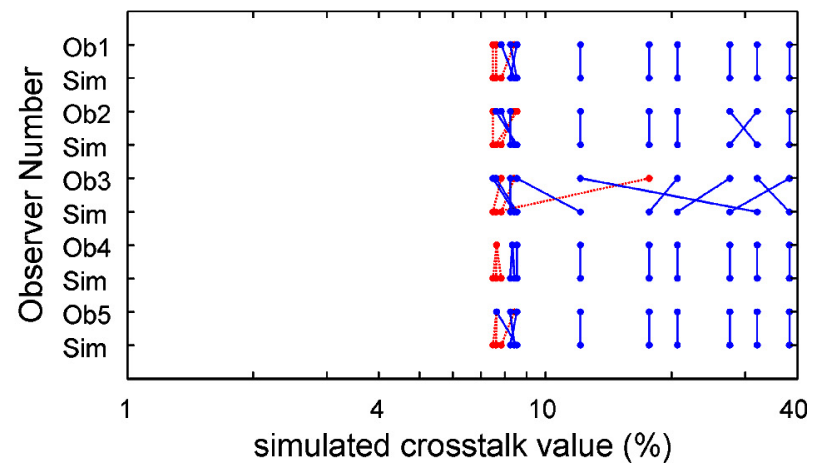

FIGURE 5 - The results of the cross-talk visual validation experiment compared to the simulated rankings. The red filter results are shown on the left column, and the cyan filter results on the right. The results for each display are plotted per row. The ranking results for each of the five observers are each plotted against the corresponding simulated ranking. The ranking of the commercial glasses are indicated in dashed red. 
TABLE 5 - Results of the statistical analysis of the visual ranking results. The table shows the correlation data for each of the display, observer, and filter-color combinations, and also the average correlation for each observer using the Spearman's rank correlation $\left(r_{\mathrm{s}}\right)$ technique as described in Sec. 4.5. (1 indicates good agreement, 0 indicates poor agreement).

\begin{tabular}{|c|c|c|c|c|}
\hline \multirow[b]{2}{*}{ Display ID } & \multirow[b]{2}{*}{ Observer } & \multicolumn{3}{|c|}{$r_{s}$ of ranking results } \\
\hline & & $\begin{array}{c}\text { red } \\
\text { filters }\end{array}$ & $\begin{array}{l}\text { cyan } \\
\text { filters }\end{array}$ & $\begin{array}{l}\text { average } r_{s} \\
\text { for each } \\
\text { observer }\end{array}$ \\
\hline \multirow[t]{5}{*}{ LCD15 } & Ob1 & 0.991 & 0.965 & 0.977 \\
\hline & Ob2 & 0.981 & 0.986 & 0.941 \\
\hline & Ob3 & 0.876 & 0.862 & 0.849 \\
\hline & Ob4 & 0.998 & 0.984 & 0.978 \\
\hline & Ob5 & 0.968 & 0.989 & 0.947 \\
\hline \multirow[t]{5}{*}{ PDP15 } & Ob1 & 0.991 & 0.979 & \\
\hline & Ob2 & 0.930 & 0.942 & \\
\hline & Ob3 & 0.921 & 0.827 & \\
\hline & Ob4 & 0.972 & 0.965 & \\
\hline & Ob5 & 0.965 & 0.951 & \\
\hline \multirow[t]{5}{*}{ CRT30 } & Ob1 & 0.981 & 0.967 & \\
\hline & Ob2 & 0.993 & 0.851 & \\
\hline & Ob3 & 0.818 & 0.862 & \\
\hline & Ob4 & 0.977 & 0.951 & \\
\hline & Ob5 & 0.972 & 0.935 & \\
\hline \multirow[t]{5}{*}{ LEDDLP1 } & Ob1 & 0.996 & 0.944 & \\
\hline & Ob2 & 0.991 & 0.853 & \\
\hline & Ob3 & 0.979 & 0.650 & \\
\hline & Ob4 & 0.984 & 0.991 & \\
\hline & Ob5 & 0.887 & 0.908 & \\
\hline
\end{tabular}

cross-talk values for each pair of glasses. This plotting technique provides more visual emphasis on ranking errors which have greater simulated cross-talk differences than ranking errors between filters which have small simulated cross-talk differences. We believe this plotting technique allows a more useful analysis of the data.

This process is further illustrated in Table 4 for one observer, display, and filter-color-combination ranking test. The first two columns show rank order as calculated by the simulation program $v$ s. the rank order as seen by the observer. Line segments have been shown between columns 1 and 2 to illustrate the quality of the comparison. Unity separation between ranking observations has been used in these first two columns. Columns 3 and 4 change the unity spacing of the observations to a spacing corresponding to the calculated cross-talk values. The values illustrated in columns 3 and 4 are then used to generate Fig. 5 - in this specific example observer Ob2 of Fig. 5(h).

The horizontal axis of Fig. 5 is shown on a logarithmic scale because the eye has a logarithmic-like response to light. The use of a logarithmic scale also reduces the bunching of the results on the left-hand side of the plots.
In cases where the observer was unable to distinguish any difference between different filters (i.e., they looked to have the same amount of cross-talk), observers were allowed to group those glasses together. Glasses that have been grouped together by an observer are plotted with the same horizontal axis value (using the mean of the corresponding simulated cross-talk values).

The commercial glasses results are plotted in dashed red, whereas the hand-made glasses are plotted in solid blue - thus allowing the commercial glasses to be easily identified. This also highlights the better performance (lower cross-talk) of the commercial glasses.

Referring to Fig. 5, in cases where the visual ranking agrees with the simulated ranking, the line segments are vertical and do not intersect. In cases where the visual and simulated rankings disagree, there will be a cross-over of the line segments.

In general terms, the validation results, as depicted in Fig. 5, agree very well with the cross-talk simulation ranking results. Across all of the tests, a high proportion (66\%) of the observations were ranked perfectly. It can be seen from the figure that ranking errors (indicated by crossing line-segments) rarely occurred across large simulated cross-talk value differences. The vast number of ranking errors occurred between filters with very similar values of simulated crosstalk. These results are statistically analyzed in the next section.

We should note that the visual ranking tests were only conducted within each display and not between displays. The cross-talk simulation results of Table 3 and Fig. 4 do indicate that LCD 15 is expected to provide noticeably lower cross-talk than the other displays when using the commercial glasses. This scenario was tested visually using red filter 3DG88 and LCD15 could be seen to have significantly lower cross-talk than PDP15, CRT30, and LEDDLP1 as predicted by the cross-talk simulation model; however, this test was only conducted informally and hence this aspect has not been validated in this particular study.

\subsection{Statistical analysis}

The quality of agreement between the visual ranking and the simulated ranking was assessed using the Spearman's rank correlation ${ }^{35}$ technique. The Spearman's rank correlation is often used in biological statistics when one or more of the variables in a dataset consist of only ranks, as is the case with the human-visual ranking of cross-talk of anaglyph glasses as described in Sec. 4.4. The Spearman rank correlation $\left(r_{\mathrm{s}}\right)$ values were calculated for all of the visual validation observations across each display, observer, and filter color combination, and these are presented in Table 5 along with the average correlation for each observer.

The average $r_{\mathrm{s}}$ value for each observer was calculated as the mean of the eight correlation results for each observer (across four displays and two filter colors). The results of 
TABLE 6 - Simulated improvement in anaglyph cross-talk performance by the use of theoretical "brick-wall" color filters as compared to the best real-world filters tested in this study.

\begin{tabular}{|c|c|c|c|}
\hline (a) Red: & \multicolumn{2}{|c|}{$\begin{array}{l}\text { simulated crosstalk (\%) } \\
\text { (Filter ID) / [pass-band] }\end{array}$} & \\
\hline Display ID & $\begin{array}{l}\text { Best Tested } \\
\text { Red Filter }\end{array}$ & $\begin{array}{l}\text { Best 'Brick-Wall' } \\
\text { Red Filter }\end{array}$ & $\begin{array}{l}\text { improve } \\
\text { ment }\end{array}$ \\
\hline LCD15 & (3DG74) & $\begin{array}{ll}3.9 \% & \\
& {[620-700 \mathrm{~nm}]}\end{array}$ & $55 \%$ \\
\hline PDP15 & (3DG88) & $\begin{array}{ll} & \\
& {[610-700 \mathrm{~nm}]}\end{array}$ & $16 \%$ \\
\hline CRT30 & (3DG74) & [625-700nm] & $65 \%$ \\
\hline LEDDLP1 & (3DG88) & $\begin{array}{ll}19.4 \% & \\
& {[615-700 \mathrm{~nm}]}\end{array}$ & $2 \%$ \\
\hline
\end{tabular}

\begin{tabular}{|c|c|c|c|}
\hline (b) Cyan: & simulated & $\begin{array}{l}\text { crosstalk (\%) } \\
\text { (Filter ID) / [pass-band] }\end{array}$ & \\
\hline Display ID & $\begin{array}{l}\text { Best Tested } \\
\text { Cyan Filter }\end{array}$ & \begin{tabular}{|c|} 
Best 'Brick-Wall' \\
Cyan Filter
\end{tabular} & $\begin{array}{l}\text { improve } \\
\text { ment }\end{array}$ \\
\hline LCD15 & $\begin{array}{ll}1.4 \% & \\
& \text { (3DG73) } \\
\end{array}$ & $\begin{array}{ll}0.3 \% & \\
& {[400-550 \mathrm{~nm}]}\end{array}$ & $31 \%$ \\
\hline PDP15 & (3DG73) & $\begin{array}{lll}1.4 \% & \\
& {[400-555 \mathrm{~nm}]} \\
\end{array}$ & $39 \%$ \\
\hline CRT30 & (3DG73) & {$[400-550 \mathrm{~nm}]$} & $20 \%$ \\
\hline LEDDLP1 & (3DG88) & $7.2 \%$ & $4 \%$ \\
\hline
\end{tabular}

observer three differed the most from the other four observers and also differed the most from the simulated rankings.

Despite the authors' initial concern about the difficulties of validating the cross-talk simulation results using the visual validation experiment, the plots of the results (Fig. 5) and the statistical analysis (Table 5) provide a high level of confidence in the accuracy of the cross-talk simulation algorithm. It can be seen in Table 5 that $78 \%$ of the ranking tests have an $r_{\mathrm{S}}$ value of 0.9 or better, and $18 \%$ have an $r_{\mathrm{s}}$ value of 0.99 or better.

The plotting technique used in Fig. 5 provides good insight into the visual validation results. The technique works very well with this relatively small number of observers but would not work well with a larger number of observers. For a larger number of observers, it would be better to focus solely on the statistical analysis.

\section{Discussion}

Given that we have established a high level of confidence in the accuracy of the anaglyph cross-talk simulation model, we can now use the model to predict the performance of a number of anaglyph cross-talk scenarios we would not otherwise be able to physically replicate. Let us consider two such scenarios.
The first scenario is to consider the performance of a pair of anaglyph glasses which have a theoretical "brickwall" filter performance (i.e., 100\% transmission in the pass region and $0 \%$ transmission in the blocking region). It will not be possible to physically test "brick-wall" filters in reality because they only exist in theory, but we believe that these simulation results will provide an indication of the absolute limit of lowest cross-talk performance achievable by optimization of the glasses alone. Table 6 lists the simulated anaglyph cross-talk performance of the four tested displays with simulated theoretical "brick-wall" anaglyph filters shown in comparison to the best tested filters for each display. The cut-off wavelength of the "brick-wall" filters were optimized for the least cross-talk for each display at $5 \mathrm{~nm}$ intervals and are indicated within square brackets on Table 6.

The simulation results indicate that even with a perfect pair of anaglyph glasses, none of the displays were able to exhibit zero cross-talk - this is because most displays output light in out-of-band wavelengths for each of the three color channels. The average anaglyph cross-talk improvement with perfect glasses across all of the displays was only $29 \%$ - the best improvement being $65 \%$ and the least improvement was $2 \%$. The lowest cross-talk achievable with a perfect filter set was with LCD15 (3.9\% for the red channel, and $0.3 \%$ for the cyan channel) - but these results are only achievable in theory. With LEDDLP1, the lowest cross-talk achieved even with theoretically perfect glasses was particularly poor at $19.4 \%$ red and $7.2 \%$ cyan. The red channel of PDP15 also had a poor minimum cross-talk of $13.9 \%$ with perfect glasses. The simulation indicates that on CRT30 a fairly large reduction of cross-talk is achievable in the red channel using perfect glasses (65\% reduction), but the actual cross-talk amount would still be fairly high at $5.9 \%$ for that eye.

The second scenario considers the cross-talk performance of LEDDLP1. Most LEDs have fairly narrow spectral emission and very little out-of-band light output. In the case of LEDDLP1, the half-intensity-width of the red, green, and blue LEDs are 17, 35, and $24 \mathrm{~nm}$, respectively (which is good), but there is a lot of out-of-band light output, particularly in the green channel as can be seen in Fig. 3(c). The authors speculate that this out-of-band light output is due to the presence of a color-accuracy algorithm within the videoprocessing path of the display which drives the display color channels based on a mix of the color-channel inputs. Since LEDs have a very narrow spectrum, they are capable of generating very richly saturated colors, so in order for the image shown on an LED TV not to be shown with overtly rich colors it will be necessary to desaturate the image by mixing the color channels. Unfortunately, this process will be detrimental for anaglyph images because it will lead to cross-talk. The authors were unable to disable this color-mixing algorithm on LEDDLP1 using the accessible menu options, but it was possible to calculate an estimation of the three-channel color spectrum of the display as if the color-mixing process was disabled (this has been given the designation 
TABLE 7 - Comparison of the simulated cross-talk performance of LEDDLP1 with the theoretical LEDDLP2 for the three commercial anaglyph glasses.

\begin{tabular}{|c|c|c|c|c|}
\hline \multicolumn{2}{|c|}{ Crosstalk(\%) } & \multicolumn{2}{|c|}{ Display ID } & \multirow{2}{*}{$\begin{array}{c}\text { improve- } \\
\text { ment }\end{array}$} \\
\hline \multicolumn{2}{|c|}{ Glasses } & LEDDLP1 & LEDDLP2 & \\
\hline \multirow[t]{3}{*}{ 3DG88 } & red & 19.7 & 0.6 & $97 \%$ \\
\hline & cyan & 7.5 & 0.6 & $92 \%$ \\
\hline & overall & 27.2 & 1.2 & $96 \%$ \\
\hline \multirow[t]{3}{*}{ 3DG74 } & red & 20.1 & 0.9 & $96 \%$ \\
\hline & cyan & 7.8 & 1.0 & $87 \%$ \\
\hline & overall & 27.9 & 1.9 & $93 \%$ \\
\hline \multirow[t]{3}{*}{ 3DG73 } & red & 20.3 & 1.1 & $95 \%$ \\
\hline & cyan & 7.6 & 0.8 & $90 \%$ \\
\hline & overall & 27.9 & 1.9 & $93 \%$ \\
\hline
\end{tabular}

LEDDLP2) and this can be fed into the cross-talk simulation model.

The cross-talk simulation results for LEDDLP2, as shown in Table 7, are remarkable - a reduction of cross-talk by as much as $97 \%$. These simulation results indicate that if the color mixing was able to be disabled on LEDDLP1, instead of exhibiting the most cross-talk, it could be exhibiting the least cross-talk. The simulated overall cross-talk of $1.2 \%$ for LEDDLP2 using the best tested glasses (3-DG88) is $71 \%$ less than even the lowest cross-talk achievable using the theoretical "brick-wall" filters on LCD15. If this is true, it will be a notable achievement. Work will continue to physically demonstrate this result.

The results of these two simulation scenarios illustrate the advantages that cross-talk simulation can provide - not only in anaglyph 3-D displays but also other stereoscopic displays. In this case, the simulations indicate that there is significantly more scope for reduction in anaglyph cross-talk by the use of more spectrally pure displays than might be gained from further improvements to the spectral performance of anaglyph glasses.

\section{Conclusion}

This paper has presented the validation of an anaglyph cross-talk simulation model which can be used to assess the improvement of 3-D image quality of anaglyph 3-D images viewed on emissive displays.

The paper has found that hand-made anaglyph glasses can exhibit significantly worse cross-talk performance than the better commercially available anaglyph 3-D glasses. Hence, the authors recommend using good commercially available anaglyph 3-D glasses rather than hand-made glasses.

The anaglyph cross-talk simulation program has also allowed us to explore the possibilities for reducing cross-talk in anaglyph systems and has found that (a) there is significant scope for reducing cross-talk by using spectrally pure emissive displays, (b) the choice of anaglyph glasses can have a significant effect on anaglyph cross-talk levels, and (c) there is only limited scope for reducing cross-talk levels by further improvements to the anaglyph glasses (compared to existing good quality commercial anaglyph glasses).

With further refinement the anaglyph cross-talk simulation program discussed in this paper could also be used to simulate and investigate the cross-talk performance of other wavelength multiplexed 3-D techniques such as Infitec, Dolby 3D, and Panavision 3D.

\section{Acknowledgments}

The authors wish to acknowledge the support of the visualization facilities from iVEC; the collaboration of Stanley Tan, Tegan Rourke, Ka Lun Yuen, Kai Karvinen, Dean Leggo, and Jesse Helliwell on various aspects of this topic; Robert Loss, Alec Duncan, Iain Parnum, and Jesse Helliwell for their assistance with the visual validation tests; and Alec Duncan and John Merritt for their assistance with the manuscript.

\section{References}

1 R. Zone, "Good old fashion anaglyph: High tech tools revive a classic format in Spy Kids 3-D," Stereo World 29(5), 11-13, 46 (2002-2003).

2 V. C. Barber and D. A. Brett, “'Colour bombardment' - A human visual problem that interferes with the viewing of anaglyph stereo material," Scanning Electron Microscopy 2, 495-498 (1982).

3 R. Patterson et al., "Binocular rivalry and head-worn displays," Human Factors 49(6), 1083-1096 (2007).

4 A. J. Woods et al., "Characterizing crosstalk in anaglyphic stereoscopic images on LCD monitors and plasma displays," J. Soc. Info. Display 15(11), 889-898 (2007).

5 L. Lipton, "Glossary," Lenny Lipton's Blog, [online] (2009). URL: http://ennylipton.wordpress.com/2009/03/16/glossary/ Dated 16 March 2009. Accessed 19 March 2010.

6 A. J. Woods, "How are crosstalk and ghosting defined in the stereoscopic literature?” Proc. SPIE Stereoscopic Displays and Applications XXII 7863, 78630Z (2011)

7 A. J. Woods, "Understanding crosstalk in stereoscopic displays (Keynote Presentation)," Proc. Intl. Conf. on 3D Systems and Applications (3DSA), 34-44 (2010).

8 F. L. Kooi and A. Toet, "Visual comfort of binocular and 3D displays," Displays 25, 99-108 (2004).

9 R. Patterson, "Review Paper: Human factors of stereo displays: An update,” J. Soc. Info. Display 17(12), 987-996 (2009).

10 Y.-Y. Yeh and L. D. Silverstein, "Limits of fusion and depth judgment in stereoscopic color displays," Human Factors 32(1), 45-60 (1990).

11 I. Tsirlin et al., "The effect of crosstalk on the perceived depth from disparity and monocular occlusions," IEEE Trans. Broadcasting 57(2), $445-453$ (2011)

12 H. Sanftmann and D. Weiskopf, “Anaglyph stereo without ghosting," Computer Graphics Forum 30(4), 1251-1259 (2011).

13 I. Ideses and L. Yaroslavsky, "Three methods that improve the visual quality of colour anaglyphs,” J. Opt. A: Pure Appl. Opt. 7(12), 755-762 (2005).

14 A. J. Woods and C. R. Harris, "Comparing levels of crosstalk with $\mathrm{red} /$ cyan, blue/yellow, and green/magenta anaglyph 3D glasses," Proc. SPIE Stereoscopic Displays and Applications XXI 7253, 0Q1-0Q12 (2010).

15 M. A. Purnell, "Casting, replication, and anaglyph stereo imaging of microscopic detail in fossils, with examples from conodonts and other jawless vertebrates," Palaeontologia Electron. 6(2), 1-11 (2003) (see Appendix 1).

16 E. Dubois, "A projection method to generate anaglyph stereo images," IEEE Intl. Conf. Acoustics, Speech, and Signal Processing, Proc. (ICASSP '01) 3, 1661-1664 (2001).

17 W. R. Sanders and D. F. McAllister, "Producing anaglyphs from synthetic images," Proc. SPIE Stereoscopic Displays and Virtual Reality Systems X 5006, 348-358 (2003). 
18 D. F. McAllister et al., "Methods for computing color anaglyphs," Proc. SPIE Stereoscopic Displays and Applications XXI 7524, 75240S$1-75240$ S-12 (2010).

19 A. J. Woods and T. Rourke, "Ghosting in anaglyphic stereoscopic images," Proc. SPIE Stereoscopic Displays and Virtual Reality Systems XI 5291, 354-365 (2004).

20 Daleh, "Build your own 3D glasses" [online]. URL: http:// www.daleh.id.au/3d_glasses.html Accessed: 26 August 2011.

21 Various authors, "How to make 3D glasses" [online]. URL: http:// www.ehow.com/how 4455680_make-3d-glasses.html Accessed: 26 August 2011.

22 Author unknown, "How to make a pair of 3D glasses for 3D Anaglyphs" [online]. URL: http://www.haworth-village.org.uk/3d/3d-glasses.asp Accessed: 26 August 2011.

23 Author unknown, "Make your own 3-D glasses" [online]. URL: http:// paperproject.org/3dglasses.html Accessed: 26 August 2011.

24 A. Agarwal, "Make your own 3D glasses in 10 seconds," Digital Inspiration [online] (2010). URL: http://www.labnol.org/home/make-3dglasses/13776/ Dated: 2 June 2010. Accessed: 6 July 2011.

25 Various authors, "How to make your own 3D glasses," Wikihow [online]. URL: http://www.wikihow.com/Make-Your-Own-3D-Glasses Accessed: 6 July 2011

26 Various authors, "Make 3-D glasses," Wired How-to Wiki [online]. URL: http://howto.wired.com/wiki/Make_3-D_Glasses Accessed: 6 July 2011.

27 S. Miles, "How to make your own 3D glasses," Pocket-lint [online] (2009). URL: http://www.pocket-lint.com/news/27268/how-to-make3d-glasses Dated: 22 September 2009. Accessed: 6 July 2011.

28 A. Stockman and L. T. Sharpe, "Luminous energy function (2 degree, linear energy)" [online] (2007). URL: http://www.cvrl.org/cvrlfunctions.htm and http://www.cvrl.org/database/text/lum/CIE2008v2.htm Accessed: 29 July 2011.

29 A. Stockman et al., "The dependence of luminous efficiency on chromatic adaptation," J. Vision 8(16): 1, 1-26 (2008). http://journalofvision.org/8/16/1/

30 K. R. Boff and J. E. Lincoln, Engineering data compendium: Human perception and performance, AAMRL, Wright-Patterson AFB, OH, pp. 370 (1988)

31 R. Blake and R. Sekuler, Perception (5th edn.) (McGraw Hill, Boston, 2006), pp. 92.

32 S. S. Stevens, "On the psychophysical law," Psychological Rev. 64(3), 153-181 (1957).

33 Z. Xie and T. G. Stockham Jr., "Toward the unification of three visual laws and two visual models in brightness perception," IEEE Trans. Systems, Man and Cybernetics 19(2), 379-387 (1989).

34 R. E. Walpole and R. H. Myers, Probability and Statistics for Engineers and Scientists (Collier Macmillan, 1985), pp. 347.

35 J. H. McDonald, Handbook of Biological Statistics (2nd edn.) (Sparky House Publishing, Baltimore, Maryland, 2009), pp. 221-223. http://udel.edu/ mcdonald/statspearman.html

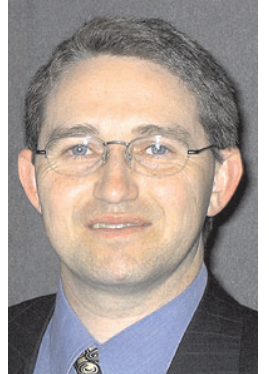

Andrew J. Woods is a research engineer at Curtin University's Centre for Marine Science \& Technology in Perth, Australia. He has MEng and BEng (Hons1) degrees in electronic engineering. He has expertise in the design, application, and evaluation of stereoscopic imaging systems for industrial and entertainment applications. He has served as co-chair of the Stereoscopic Displays and Applications conference since 2000.

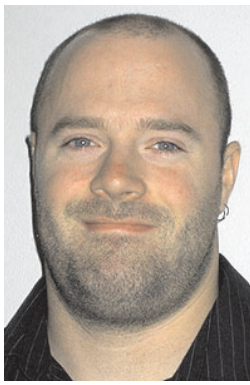

Chris R. Harris is a graduate of Curtin University with a B.S. degree in applied physics and has interests in electronics and information technology. He is currently employed by Murdoch University. 